\title{
Inclusive electron scattering and the GENIE neutrino event generator
}

\author{
A. Papadopoulou $\odot,{ }^{1}$ A. Ashkenazi, ${ }^{1, *}$ S. Gardiner $\odot,{ }^{2}$ M. Betancourt, ${ }^{2}$ S. Dytman $\odot{ }^{3}$ L. B. Weinstein, ${ }^{4}$ \\ E. Piasetzky, ${ }^{5}$ F. Hauenstein, ${ }^{4,1}$ M. Khachatryan, ${ }^{4}$ S. Dolan, ${ }^{6}$ G. D. Megias $\odot,{ }^{7,8}$ and O. Hen ${ }^{1}$ \\ (e4v Collaboration) \\ ${ }^{1}$ Massachusetts Institute of Technology, Cambridge, Massachusetts 02139, USA \\ ${ }^{2}$ Fermi National Accelerator Laboratory, Batavia, Illinois 60510, USA \\ ${ }^{3}$ University of Pittsburgh, Pittsburgh, Pennsylvania 15260, USA \\ ${ }^{4}$ Old Dominion University, Norfolk, Virginia 23529, USA \\ ${ }^{5}$ School of Physics and Astronomy, Tel Aviv University, Tel Aviv 69978, Israel \\ ${ }^{6}$ CERN, European Organization for Nuclear Research, Geneva 23, Switzerland \\ ${ }^{7}$ Research Center for Cosmic Neutrinos, Institute for Cosmic Ray Research, \\ University of Tokyo, Kashiwa, Chiba 277-8582, Japan \\ ${ }^{8}$ Departamento de Fisica Atomica, Molecular y Nuclear, Universidad de Sevilla, 41080 Sevilla, Spain
}

(Received 30 September 2020; revised 14 April 2021; accepted 30 April 2021; published 8 June 2021)

The extraction of neutrino mixing parameters from accelerator-based neutrino-oscillation experiments relies on proper modeling of neutrino-nucleus scattering processes using neutrino interaction event generators. Experimental tests of these generators are difficult due to the broad range of neutrino energies produced in accelerator-based beams and the low statistics of current experiments. Here we overcome these difficulties by exploiting the similarity of neutrino and electron interactions with nuclei to test neutrino event generators using high-precision inclusive electron-scattering data. To this end, we revised the electron-scattering mode of the GENIE event generator ( $e$-GENIE) to include electron-nucleus bremsstrahlung radiation effects and to use, when relevant, the exact same physics models and model parameters, as the standard neutrino-scattering version. We also implemented new models for quasielastic (QE) scattering and meson exchange currents (MECs) based on the theory-inspired super scaling approach SuSAv2. Comparing the new $e$-GENIE predictions with inclusive electron-scattering data, we find an overall adequate description of the data in the QE- and MEC-dominated lower energy transfer regime, especially when using the SuSAv2 models. Higher energy transfer interactions, which are dominated by resonance production, are still not well modeled by $e$-GENIE.

DOI: $10.1103 /$ PhysRevD.103.113003

\section{INTRODUCTION}

The extraction of neutrino mixing parameters from neutrino-oscillation experiments [1-3] relies on comparing the energy-dependent neutrino event distribution for a particular neutrino flavor near the neutrino production point with that at a significant distance away. In practice, the yield at each neutrino energy is extracted from the measured neutrino-nucleus interactions in a detector, as reconstructed from the measured particles ejected in the

\footnotetext{
* Corresponding author. adishka@mit.edu

Published by the American Physical Society under the terms of the Creative Commons Attribution 4.0 International license. Further distribution of this work must maintain attribution to the author(s) and the published article's title, journal citation, and DOI.
}

neutrino-nucleus interaction. This requires detailed knowledge of the $\nu$-nucleus interaction.

Unfortunately, measuring the $\nu$-nucleus interaction is difficult due to the wide-energy spread of acceleratorproduced neutrino beams [see, e.g., Fig. 1 (left)] and the tiny $\nu$-nucleus cross section. A relatively small body of data has been published [4], which suffers from poor statistics and is flux averaged over a wide range of neutrino energies. These data are then supplemented with theoretical models and implemented into event generator codes such as GENIE [5] to simulate the $\nu$-nucleus interaction across a wide range of energies and targets. GENIE simulations are then used to aid in extraction of the incident neutrino flux as a function of energy from the $\nu$-nucleus scattering events measured in neutrino detectors.

However, the theoretical models need to describe many different interaction processes for medium to heavy nuclei 


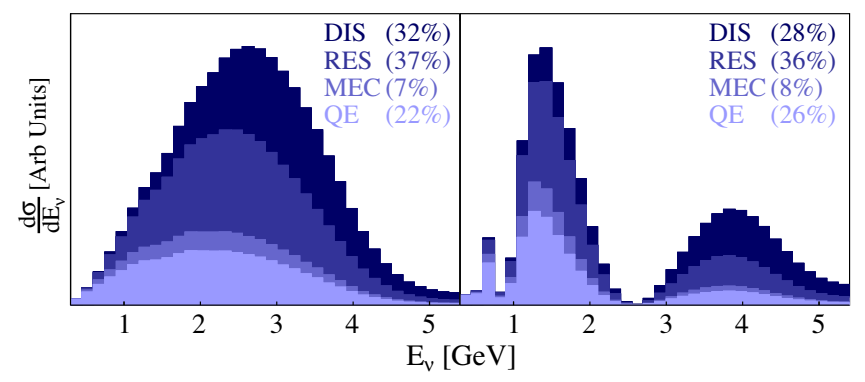

FIG. 1. Charged-current cross sections as a function of neutrino energy obtained using GENIE for muon neutrino scattering using the DUNE near detector (left) and far detector (right) oscillated fluxes [6]. The shaded bands show the fractional contribution for each interaction mechanism, quasielastic (QE) scattering, mesonexchange currents (MECs), resonance excitation (RES), and deep inelastic scattering (DIS). See text for details of the interaction mechanisms. The numbers in parentheses indicate the percentage of the cross section due to each interaction mechanism.

(typically $\mathrm{C}, \mathrm{O}$, or Ar) where nuclear effects complicate the interactions. As a result, the uncertainties in the extraction of oscillation parameters are often dominated by lack of knowledge of the $\nu$-nucleus interaction [1,2].

Figure 1 shows such a wide-energy spectrum for the DUNE near detector flux-averaged cross sections (left) and the far detector oscillated flux-averaged cross sections (right) using one model configuration in GENIE. All four $\nu$-nucleus reaction mechanisms contribute significantly and all four need to be well understood. This is especially true because different reaction mechanisms contribute differently in the different oscillation peaks. Understanding one reaction mechanism better than the others could have significant oscillation-analysis implications.

Because neutrinos and electrons are both leptons, they interact with atomic nuclei in similar ways (see Fig. 2). Electrons interact via a vector current $\left(j_{\mathrm{EM}}^{\mu}=\bar{u} \gamma^{\mu} u\right)$ and neutrinos interact via vector and axial-vector $\left(j_{C C}^{\mu}=\right.$ $\left.\bar{u} \gamma^{\mu}\left(1-\gamma^{5}\right) u \frac{-i g_{W}}{2 \sqrt{2}}\right)$ currents.

This gives an inclusive $\left(e, e^{\prime}\right)$ electron-nucleonscattering cross section that depends on only two structure functions,
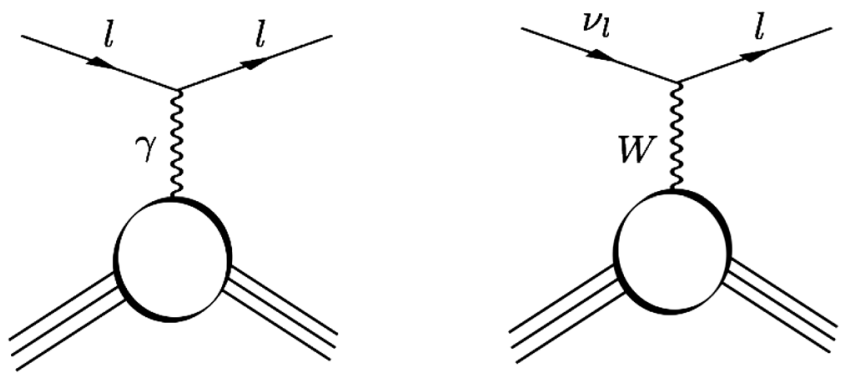

FIG. 2. Left: electron-nucleus inclusive scattering via onephoton exchange. Right: charged-current neutrino-nucleus inclusive scattering via $W$ exchange with a final-state charged lepton.

$$
\frac{d^{2} \sigma^{e}}{d x d Q^{2}}=\frac{4 \pi \alpha^{2}}{Q^{4}}\left[\frac{1-y}{x} F_{2}^{e}\left(x, Q^{2}\right)+y^{2} F_{1}^{e}\left(x, Q^{2}\right)\right] .
$$

Here $F_{1}^{e}$ and $F_{2}^{e}$ are the standard electromagnetic vector structure functions, $Q^{2}=\mathbf{q}^{2}-\omega^{2}$ is the squared momentum transfer, and $\mathbf{q}$ and $\omega$ are the three-momentum and energy transfers, $x=Q^{2} /(2 m \omega)$ is the Bjorken scaling variable, $m$ is the nucleon mass, $y=\omega / E_{e}$ is the electron fractional energy loss, and $\alpha$ is the fine structure constant. This formula is valid for $Q^{2} \gg m^{2}$ where the electronnucleon cross section is simplest. Cross sections at lower $Q^{2}$ have more complicated factors multiplying each of the two structure functions. We show the simpler form to make the electron-neutrino cross section correspondence more clear.

The corresponding inclusive charged-current (CC) $\left(\nu, l^{ \pm}\right)$neutrino-nucleon cross section (where $l^{ \pm}$is the outgoing charged lepton) has a similar form with the addition of a third axial structure function,

$$
\begin{aligned}
\frac{d^{2} \sigma^{\nu}}{d x d Q^{2}}= & \frac{G_{F}^{2}}{2 \pi}\left[\frac{1-y}{x} F_{2}^{\nu}\left(x, Q^{2}\right)+y^{2} F_{1}^{\nu}\left(x, Q^{2}\right)\right. \\
& \left.-y(1-y / 2) F_{3}^{\nu}\left(x, Q^{2}\right)\right] .
\end{aligned}
$$

Here $F_{1}^{\nu}$ and $F_{2}^{\nu}$ are parity-conserving structure functions, $F_{3}^{\nu}$ is a new parity-violating structure function, and $G_{F}$ is the Fermi constant. The parity-conserving structure functions $F_{1}^{\nu}$ and $F_{2}^{\nu}$ both include a vector-vector term almost identical to $F_{1}^{e}$ and $F_{2}^{e}$ (the electron terms have both isoscalar and isovector components, but the neutrino terms have only isovector components) and an additional axialaxial term. See Refs. $[4,7,8]$ for more detail.

These simple equations are very similar for leptonnucleus scattering. In the limit of electron-nucleon elastic scattering $(x=1)$, the two structure functions reduce to the Dirac and Pauli form factors [which are linear combinations of the electric and magnetic form factors, $G_{E}\left(Q^{2}\right)$ and $G_{M}\left(Q^{2}\right)$ ]. Neutrino-nucleon elastic scattering has an additional axial form factor. In the simplest case where a lepton scatters quasielastically from a nucleon in the nucleus and the nucleon does not reinteract as it leaves the nucleus, then the lepton-nucleus cross section is the integral over all initial-state nucleons,

$$
\begin{aligned}
\frac{d \sigma}{d E d \Omega}= & \int_{\mathbf{p}_{\mathbf{i}}} \int_{E_{b}} d^{3} \mathbf{p}_{\mathbf{i}} d E_{b} K S\left(\mathbf{p}_{\mathbf{i}}, E_{b}\right) \frac{d \sigma^{\mathrm{free}}}{d \Omega} \\
& \times \delta^{3}\left(\mathbf{q}-\mathbf{p}_{\mathbf{f}}-\mathbf{p}_{\mathbf{r}}\right) \delta\left(\omega-E_{b}-T_{f}-T_{r}\right),
\end{aligned}
$$

where $\mathbf{p}_{\mathbf{i}}$ and $\mathbf{p}_{\mathbf{f}}$ are the initial and final momenta of the struck nucleon (in the absence of reinteraction, $\left.\mathbf{p}_{\mathbf{f}}=\mathbf{q}+\mathbf{p}_{\mathbf{i}}\right), \mathbf{p}_{\mathbf{r}}=-\mathbf{p}_{\mathbf{i}}$ is the momentum of the recoil $A-1$ nucleus, $E_{b}$ is the nucleon binding energy, $S\left(\mathbf{p}_{\mathbf{i}}, E_{b}\right)$ 
is the probability of finding a nucleon in the nucleus with momentum $\mathbf{p}_{\mathbf{i}}$ and binding energy $E_{b}, T_{f}$ and $T_{r}$ are the kinetic energies of the final-state nucleon and $A-1$ system, $d \sigma^{\text {free }} / d \Omega$ is the lepton-bound nucleon elastic cross section, and $K$ is a known kinematic factor.

This simple form is complicated by nucleon reinteraction which changes the overlap integral between the initial and final states (and thus the cross section) and changes the momentum and angle of the outgoing nucleon.

Thus, to calculate even the simplest type of leptonnucleus interaction, we need to know the momentum and binding energy distribution of all nucleons in the nucleus, how the outgoing nucleon wave function is distorted by the nucleon-nucleus potential, and how the outgoing nucleon kinematics is changed by final-state interactions. The initial nuclear state is believed to be identical for $e$ - and $\nu$-nucleus interactions. However, the details of each interaction make the final states different. For example, neutral current $\nu$-nucleus interactions are very similar to $e$-nucleus interactions because both have neutral exchanged bosons $\left(\gamma\right.$ and $\left.Z^{0}\right)$. However, the couplings and form factors will be different. Charged-current $\nu$-nucleus interactions are different because the charged exchanged boson $\left(W^{ \pm}\right)$ changes the final-state total hadronic charge by \pm 1 .

In addition, the lepton can knock out two nucleons simultaneously, either by interacting with a nucleon belonging to a short range correlated (SRC) pair [9] or by interacting with a pair of nucleons correlated via MECs [10]. And, of course, these two interactions add coherently. The lepton can interact with a nucleon, exciting it to a resonance, which then deexcites, typically resulting in the emission of a nucleon plus mesons. The lepton can also scatter inelastically from a quark in a nucleon (DIS). The outgoing hadrons in all of these interactions will interact identically with the residual nucleus, whether they are knocked out by an electron or by a neutrino.

MECs are relatively poorly understood and this contributes significantly to neutrino-oscillation uncertainties. References [11,12] showed that inclusive neutrino MEC cross sections can be calculated directly from the structure functions in their electron-scattering counterparts if the interacting system is nonrelativistic and if only transverse response functions (i.e., those which concern the spatial components of the current transverse to the direction of momentum transfer) contribute to the cross section. This latter assumption is justified for electron MEC interactions by microscopic studies [11] and electron-scattering data analyses. Its application to neutrino-scattering data via the GiBUU theory framework [13] provides favorable comparisons in $2 p 2 h$-enhanced regions [14]. However, the same microscopic model applied to neutrino scattering suggests that, while the transverse component generally remains dominant, the axial component of the longitudinal response function can become important (especially for antineutrinos), breaking the direct link between electron and neutrino
MEC interactions [15]. Despite these shortcomings, electron scattering can still provide crucial inputs to modeling neutrino MEC interactions.

Electron-nucleus scattering is much easier to understand than $\nu$-nucleus scattering for three reasons:

(i) Electron beams have a single, well-known energy.

(ii) Electron experiments have far less statistical uncertainty because electron beams have higher flux and $e$-nucleus cross sections are far higher than their $\nu$ counterparts.

(iii) Electron cross sections are purely vector.

Therefore we can use $e$-nucleus scattering to constrain models of $\nu$-nucleus scattering. Any model which fails to accurately describe $e A$ (vector-vector) scattering data cannot be used with confidence to simulate $\nu A$ (vectorvector + axial-axial + vector-axial) interactions.

GENIE started as a neutrino event generator, like almost all event generators in neutrino physics. In recognition of the importance of electron scattering, it was added as a new option in close conjunction with the neutrino scattering section. As much as possible, the neutrino section references vector and axial contributions separately and uses the same modeling for vector interactions as the electron section. Some models were developed separately for electrons and others were developed for both applications in tandem.

An earlier electron version of GENIE (v2.12) was tested in Ref. [16] by comparing with inclusive $\left(e, e^{\prime}\right)$ data. Although the quasielastic peak was well described for a variety of energies and nuclei, the resonance region (total hadronic energy $W>1.1 \mathrm{GeV}$ ) was poorly described. However, the establishment of full compatibility between the electron and neutrino versions was then still in its early stages.

Here we significantly improved both neutrino and electron versions of GENIE to address these and other issues. We fixed significant errors in the previous version, including an error in the Mott cross section in the electron QE Rosenbluth interaction, a missing Lorentz boost in the MEC interaction (for both $e$ and $\nu$ interactions), and incorrect electron couplings used in the RES interactions. We worked to better integrate the electron and neutrino codes for quasielastic and $2 p 2 h$ models. We also added more up-to-date models such as the super scaling approach SuSAv2 [8]. These changes have been incorporated in the latest GENIE version. We refer to the electron-scattering component of the widely used GENIE [5] event generator as $e$-GENIE.

The GENIE improvements can be seen in Fig. 3. The QE peak (at $\omega \approx 0.15$ ) predicted by the older GENIE $\mathrm{v} 2$ is too large and is slightly shifted to higher energy transfer than the data and the first resonance peak is at much too large an energy transfer. The QE peak predicted by the updated GENIE v3 has about the correct integral and is at the correct energy transfer (but is slightly too narrow) and the first resonance peak is located at $m_{\Delta}-m \approx 300 \mathrm{MeV}$ beyond 


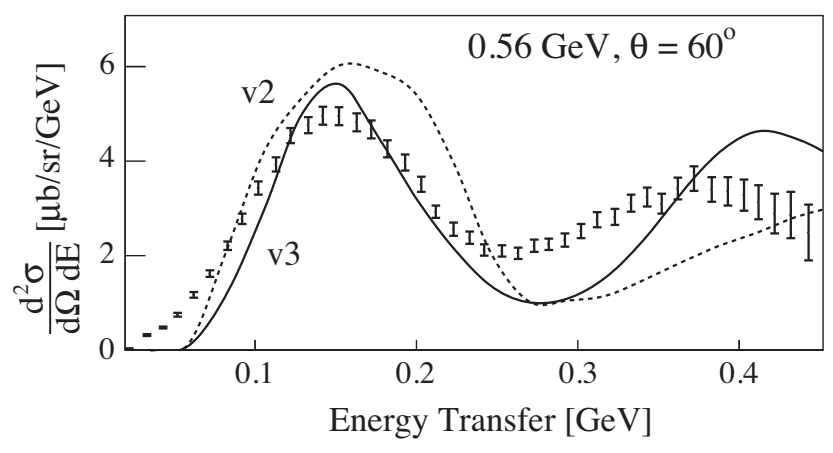

FIG. 3. Comparison between GENIE v2 and v3 descriptions of inclusive $\mathrm{C}\left(e, e^{\prime}\right)$ scattering cross sections at $E_{0}=0.56 \mathrm{GeV}$, $\theta_{e}=60^{\circ}$, and $Q_{Q E}^{2} \approx 0.24 \mathrm{GeV}^{2}$ [17]. Black points show the data, solid black line shows the GENIE v3 results, and dashed black line shows the GENIE v2 results.

the QE peak, as expected. Details of the calculations and of the discrepancies between GENIE v3 and the data are discussed in detail below.

We specifically focus on testing our knowledge of the electron-nucleus cross section by benchmarking $e$-GENIE against existing inclusive electron scattering data for different target nuclei, beam energies, and scattering angles. The goals are very similar to Ref. [16], but we test a much more modern version of $e$-GENIE and we also compare different models within $e$-GENIE. In addition, if $e$-GENIE describes electron-nucleus scattering well, then it would be an improvement on the former empirical fit [18] and would be valuable for helping simulate a variety of electron experiments.

\section{MODELING}

The most common lepton-nucleus interaction mechanisms include (Fig. 4) (a) QE scattering from individual moving nucleons in the nucleus, (b) two-nucleon knockout, due to interactions with a meson being exchanged between two nucleons (referred to as two-particle two-hole excitations $2 p 2 h$ or its major component, MECs), (c) interactions which leave the struck nucleon in an excited state (resonance production or RES), and (d) nonresonant interactions with a quark within the nucleon (DIS).
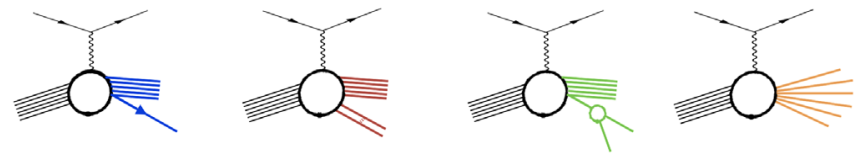

FIG. 4. Reaction mechanisms for lepton-nucleus scattering. (a) QE scattering where one nucleon is knocked out of the nucleus, (b) $2 p 2 h$ (MEC) where two nucleons are knocked out of the nucleus, (c) resonance production where a nucleon is excited to a resonance which decays to a nucleon plus meson(s), and (d) DIS where the lepton interacts with a quark in the nucleon.
However, GENIE does not include interference between the amplitudes of different reaction modes; i.e., the total cross section is obtained by adding the individual cross sections $\sigma_{i}(E)$ incoherently.

For fixed incident beam energy and scattered electron angle, the dominant process changes from $\mathrm{QE}$ at low energy transfer $\left(\omega \approx Q^{2} / 2 m\right)$ through MECs to RES and to DIS at high energy transfer. Therefore, examining the agreement of $e$-GENIE with data as a function of energy transfer can provide valuable insight into the specific shortcomings of the $e$-GENIE models and their implementations. This separation according to the underlying physics interactions gives valuable insights which are not presently possible with neutrino cross sections, because only broadenergy beams are available.

The GENIE simulation framework offers several models of the nuclear ground state, several models for each of the $e A$ or $\nu A$ scattering mechanisms (each with various tunable model parameters), and several models for hadronic finalstate interactions (FSIs), i.e., intranuclear rescattering of the outgoing hadrons $[5,19,20]$. In this section, we describe the different models relevant for this work and the electronspecific effects that we accounted for during $e$-GENIE development.

Since our goal is to use electron-scattering data to validate neutrino-interaction modeling in GENIE, the GENIE code for electron and neutrino interactions are unified in many places. The neutrino interacts with a nucleus via the weak interaction and massive $W$ or $Z$ exchange, whereas the electron interacts mostly electromagnetically via massless photon exchange, see Fig. 2. This causes the cross sections to differ by an overall factor of

$$
\frac{8 \pi^{2} \alpha^{2}}{G_{F}^{2}} \frac{1}{Q^{4}}
$$

[see Eqs. (1) and (2)]. In the code, both interactions use the same nuclear ground state and many of the nuclear reaction effects (e.g., FSIs) are very similar or identical. Except for mass effects and form factors, the electron-nucleus cross section can be obtained by setting the axial part of the interaction to zero. We also accounted for isoscalar and isovector terms appropriately.

Many of the models reported in this work (except for SuSAv2) use the GENIE implementation of the local Fermi gas (LFG) model to describe the nuclear ground state. In the simplest Fermi gas model, nucleons occupy all momentum states up to the global Fermi momentum $k_{F}$ with equal probability. In the LFG model, the Fermi momentum at a given radial position depends on the local nuclear density (obtained from measurements of nuclear charge densities). To account for this radial dependence, GENIE selects an initial momentum for the struck nucleon by first sampling an interaction location $r$ inside the nucleus according to the nuclear density. The nucleon momentum 
is then drawn from a Fermi distribution using the local Fermi momentum $k_{F}(r)$.

Another commonly used nuclear model is the relativistic Fermi gas (RFG). Here a global momentum distribution is used for the entire nucleus, independent of the interaction location in the nucleus. However, a high-momentum tail of nucleons with momenta above the Fermi momentum is included. This tail is meant to approximately account for the effects of two-nucleon short-range correlations $[9,21]$ and follows a $1 / k^{4}$ distribution, where $k$ is the nucleon momentum.

We consider two distinct sets of GENIE models for QE and MECs:

(i) The G2018 model set, which uses the Rosenbluth cross section with the local Fermi gas for QE scattering and the empirical MEC model [22], is formally marked as the G18_10a_02_11a configuration of GENIE $v 3$.

(ii) GSuSAv2 [23], which follows the universal SuSAv2 superscaling approach to lepton scattering, is a new model set that will be included in the forthcoming GENIE v3.2.0 release as the GTEST19_10b_00_000 configuration.

In both model sets, RES is modeled using the BergerSehgal model [24] and DIS reactions are modeled using Bodek and Yang [25]. The models are described in more detail below.

\section{A. Quasielastic}

In $\mathrm{QE}$ interactions, a lepton scatters on a single nucleon, removing it from the spectator $A-1$ nucleus unless finalstate interactions lead to reabsorption.

The electron QE interaction in the G2018 configuration of GENIE uses the Rosenbluth cross section with the vector structure function parametrization of Ref. [26]. We corrected the implementation of this model for $e$-GENIE and modified the cross section as described above. This electron QE cross section differs in important ways (notably, the Rosenbluth treatment lacks medium polarization corrections) from the Valencia CCQE model [27] used in the G2018 configuration for neutrinos.

A new QE model in GENIE, based on the SuSAv2 approach $[15,23,28]$, uses superscaling to write the inclusive cross section in terms of a universal function (i.e., independent of momentum transfer and nucleus). For electron scattering, the scaling function may be expressed in the form

$$
f\left(\psi^{\prime}\right)=k_{F} \frac{\frac{d^{2} \sigma}{d \Omega_{e} d \nu}}{\sigma_{\operatorname{Mott}}\left(v_{L} G_{L}^{e e^{\prime}}+V_{T} G_{T}^{e e^{\prime}}\right)},
$$

where $\psi^{\prime}$ is a dimensionless scaling variable, $k_{F}$ is the nuclear Fermi momentum, the denominator is the singlenucleon elastic cross section, $v_{L}$ and $v_{T}$ are known functions of kinematic variables, and $G_{L}^{e e^{\prime}}(q, \omega)$ and $G_{T}^{e e^{\prime}}(q, \omega)$ are the longitudinal and transverse nucleon structure functions (linearly related to $F_{1}^{e}$ and $F_{2}^{e}$ ) [29]. For $e$-GENIE, we extended the original neutrino implementation [23] to the electron case.

The original SuSAv2 QE cross section calculations used a relativistic mean field (RMF) model of the nuclear ground state $[30,31]$. This approach includes the effects of the real part of the nucleon-nucleus potential on the outgoing nucleons which creates a "distorted" nucleon momentum distribution.

Although GENIE lacks the option to use a RMF nuclear model directly, we achieve approximate consistency with the RMF-based results by using a two-step strategy for QE event generation. First, an energy and scattering angle for the outgoing lepton are sampled according to the inclusive double-differential cross section. This cross section is computed by interpolating precomputed values of the nuclear responses $G_{L}^{e e^{\prime}}(q, \omega)$ and $G_{L}^{e e^{\prime}}(q, \omega)$ which are tabulated on a two-dimensional grid in $(q, \omega)$ space. The responses were obtained using the original RMF-based SuSAv2 calculation.

Second, the outgoing nucleon kinematics are determined by choosing its initial momentum from a LFG distribution. The default nucleon binding energy used in GENIE for the LFG model is replaced for SuSAv2 with an effective value tuned to most closely duplicate the RMF distribution. The outgoing nucleon kinematics are not needed for the comparisons to inclusive $\left(e, e^{\prime}\right)$ data shown in this work.

We also compared those models for QE scattering to a model using the Llewellyn-Smith CCQE scattering prescription [32] and the RFG.

\section{B. Meson-exchange current}

MECs describe an interaction that results in the ejection of two nucleons from the nucleus (often referred to as $2 p 2 h$ ). It typically proceeds via lepton interaction with a pion being exchanged between two nucleons or by interaction with a nucleon in a SRC pair. MECs are far less understood than other reaction mechanisms because, unlike the others, it involves scattering from two nucleons simultaneously. GENIE has several models for MECs.

The G2018 configuration of $e$-GENIE uses the empirical model [22], that is useable for both $e A$ and $\nu A$ scattering. It assumes that the MEC peak for inclusive scattering has a Gaussian distribution in $W$ and is located between the QE and first RES peaks. Although both versions of the model use the same effective form factors, the amplitude of the MEC peak was tuned separately to electron and neutrinoscattering data. This model was developed in the context of empirically fitting GENIE to MiniBooNE inclusive neutrino-scattering data and is still used for neutral-current interactions. 
For charged-current neutrino interactions, GENIE G2018 uses the very different Valencia $2 p 2 h$ model $[27,33]$ instead of the empirical model.

The SuSAv2 model evaluates the $2 p 2 h$ MEC contributions within an exact RFG-based microscopic calculation that englobes the $2 p 2 h$ states excited by the action of meson-exchange currents within a fully relativistic framework $[11,15,34,35]$ and considers the weak vector and axial components for neutrino-nucleus interactions in both longitudinal and transverse channels as well as a complete analysis for electromagnetic reactions. As in the case for the SuSAv2 QE model, we extended the original GENIE implementation of SuSAv2 MEC for neutrinos [23] to the electron case for $e$-GENIE. The SuSAv2 MEC model is available for both $e A$ and $\nu A$ scattering $[28,36,37]$.

\section{Resonance and deep inelastic scattering}

Resonance production in GENIE is simulated using the Berger-Sehgal model [24], in which the lepton interacts with a single moving nucleon and excites it to 1 of 16 resonances. The cross sections are calculated based on the Feynman-Kislinger-Ravndal model [38], without any interferences between them. Form factors are derived separately for vector and axial probes [39] but have not been updated to include recent electron-scattering results.

The GENIE treatment of deep inelastic scattering used in this work is based on that of Bodek and Yang [25]. Hadronization is modeled using an approach which transitions gradually between the Andreopoulos-GallagherKehayias-Yang model [40] and the PYTHIA 6 model [41]. At low values of the hadronic invariant mass $W$, the BodekYang differential cross section is scaled by tunable parameters that depend on the multiplicity of hadrons in the final state [20].

Integration of RES and DIS contributions is complicated by the need for a model of nonresonant meson production. There is no definite separation of RES and DIS contributions; GENIE makes a sharp cutoff at $W=1.93 \mathrm{GeV}$ in the latest tune and uses a suppression factor to enable usage of the Bodek-Yang cross section at low $W$ in place of a true nonresonant model. These features were recently retuned by the GENIE Collaboration using measurements of charged-current $\nu_{\mu}$ and $\bar{\nu}_{\mu}$ scattering on deuterium [42]. The $W$ cutoff and suppression factors apply to both $e A$ and $\nu A$ models.

\section{FINAL-STATE INTERACTIONS}

Final-state interactions of outgoing with hadrons with the residual nuclei are calculated in $e$-GENIE using the INTRANUKE $[19,43]$ package and one of two options. The first, hA, an empirical data-driven method, uses the cross section of pions and nucleons with nuclei as a function of energy up to $1.2 \mathrm{GeV}$ and the CEM03 [44] calculation for higher energies. The second, $\mathrm{hN}$, is a full intranuclear cascade calculation of the interactions of pions, kaons, photons, and nucleons with nuclei. In the $\mathrm{hN}$ model, each outgoing particle can interact successively with any or all the nucleons it encounters on its path leaving the nucleus, and any particles created in those interactions can also subsequently reinteract. The ability of the two models to describe hadron-nucleus data is very similar.

The $e$-GENIE G2018 configuration uses the hA FSI model, while GSuSAv2 uses hN. However, the choice of FSI model has no effect on the inclusive cross sections considered in the present work.

\section{RADIATIVE CORRECTIONS}

When electrons scatter from nuclei, there are several radiative effects that change the cross section. The incoming and outgoing electrons can each radiate a real photon, which changes the kinematics of the interaction or the detected particles, and there can be vertex or propagator corrections that change the cross section. When comparing electron-scattering data to models, either the data or the model needs to be corrected for radiative effects. Published electron-scattering cross sections are typically corrected for radiative effects, but this correction is complicated and somewhat model dependent.

We implemented a framework for electron radiative corrections in GENIE for the first time to allow comparisons to nonradiatively corrected data. The framework allows electron radiation, which can change the kinematics of the event by changing either the incident or scattered electron energy (through radiation of a real photon). We modeled external radiation in the same way as the Jefferson Lab SIMC event generator [45]. Future versions of $e$-GENIE will incorporate cross section changes due to vertex and propagator corrections.

We validated the radiative correction procedure by comparing a simulated sample to electron scattering from protons at Jefferson Lab. Figure 5 shows the data compared to the GENIE simulation with and without radiative corrections. The radiatively corrected calculation is clearly much closer to the data.

This correction can be used for comparisons with nonradiatively corrected data. It was not used to compare with the radiatively corrected inclusive data shown below.

\section{V. $e$-GENIE COMPARISONS TO INCLUSIVE ELECTRON-SCATTERING DATA}

To test $e$-GENIE, we compare inclusive electron-scattering data to theoretical predictions made using two different program configurations which differ in their choice of $\mathrm{QE}$ and MEC models: G2018 (which adopts the Rosenbluth model for QE and the empirical Dytman model for MECs) and GSuSAv2 (which adopts SuSAv2 for both QE and MECs). 


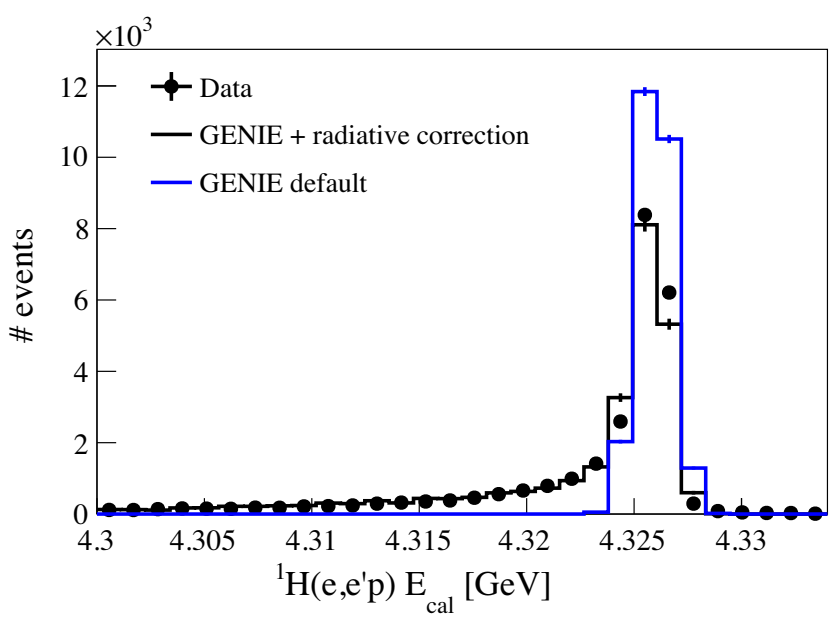

FIG. 5. Number of events vs $E_{\text {cal }}=E_{e^{\prime}}+T_{p}$ the scattered electron energy plus proton kinetic energy for $4.32 \mathrm{GeV}$ $\mathrm{H}\left(e, e^{\prime} p\right)$. Black points are data [46], black histogram shows the unradiated GENIE prediction, and blue histogram shows the GENIE prediction with electron radiation. The GENIE calculations have been scaled to have the same integral as the data.

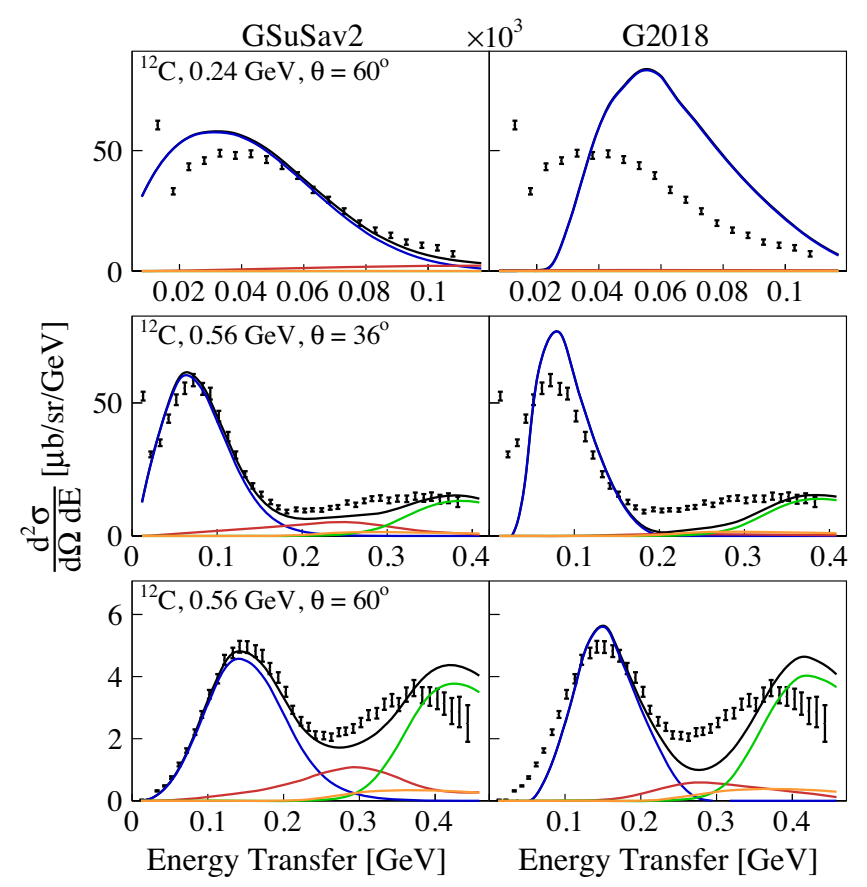

FIG. 6. Comparison of inclusive $\mathrm{C}\left(e, e^{\prime}\right)$ scattering cross sections for data and for GENIE. Left: data vs GSuSAv2. Right: data vs G2018. Top: $E_{0}=0.24 \mathrm{GeV}, \theta_{e}=60^{\circ}$, and $Q_{\mathrm{QE}}^{2} \approx 0.05 \mathrm{GeV}^{2}$ [17]. Middle: $E_{0}=0.56 \mathrm{GeV}, \theta_{e}=36^{\circ}$, and $Q_{\mathrm{QE}}^{2} \approx 0.11 \mathrm{GeV}^{2}$ [17]. Bottom: $E_{0}=0.56 \mathrm{GeV}, \theta_{e}=60^{\circ}$, and $Q_{\mathrm{QE}}^{2} \approx 0.24 \mathrm{GeV}^{2}$ [17]. Black points show the data, solid black lines show the total GENIE prediction, colored lines show the contribution of the different reaction mechanisms: blue, QE; red, MEC; green, RES; and orange, DIS.
Figures 6-8 show the inclusive $\mathrm{C}\left(e, e^{\prime}\right)$ cross sections for a wide range of beam energies and scattering angles compared to the G2018 and GSuSAv2 models. The QE peak is the one at lowest energy transfer $\left(\nu \approx Q^{2} / 2 m\right)$ in each plot. The next peak at about $300 \mathrm{MeV}$ larger energy transfer corresponds to $\Delta(1232)$ excitation and the "dip region" is between the two peaks. The $\Delta$ peak in the data is separated from the QE peak by less than the $300 \mathrm{MeV}$ $\Delta$-nucleon mass difference, indicating that it is shifted in the nuclear medium. This shift is more visible at lower momentum transfer where the $\Delta$ peak is more prominent.

GSuSAv2 clearly describes the $\mathrm{QE}$ and dip regions much better than G2018, especially at the three lowest momentum transfers (see Fig. 6). G2018 has particular difficulty describing the data for $E_{0}=0.24 \mathrm{GeV}$ and $\theta_{e}=60^{\circ}$, where $Q^{2}=0.05 \mathrm{GeV}^{2}$ at the quasielastic peak. G2018 also predicts too small a width for the quasielastic peak and too small a $2 p 2 h / \mathrm{MEC}$ contribution for $E_{0}=0.56 \mathrm{GeV}$ and $\theta_{e}=60^{\circ}$; GSuSAv2 describes both features far better.

At intermediate momentum transfers (see Fig. 7), GSuSAv2 describes the data somewhat better than G2018, although it overpredicts the dip-region cross section at $E_{0}=1.299 \mathrm{GeV}$ and $\theta_{e}=37.5^{\circ}$. The MEC

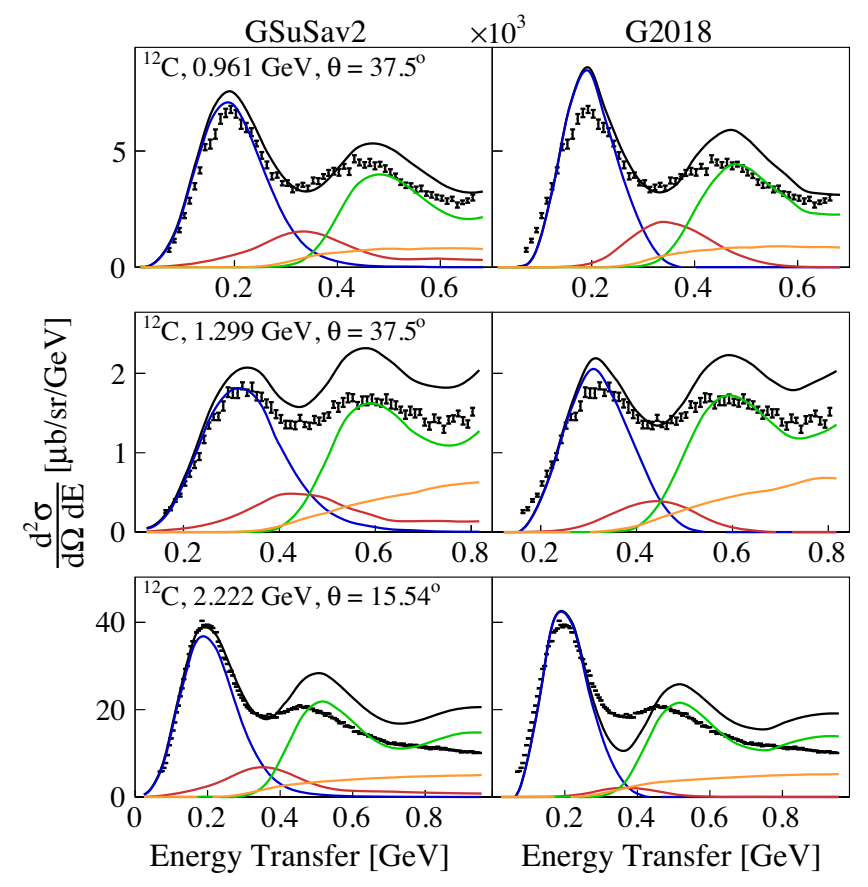

FIG. 7. Comparison of inclusive $\mathrm{C}\left(e, e^{\prime}\right)$ scattering cross sections for data and for GENIE. Left: data vs GSuSAv2. Right: data vs G2018. Top: $E_{0}=0.96 \mathrm{GeV}, \theta_{e}=37.5^{\circ}$, and $Q_{\mathrm{QE}}^{2} \approx$ $0.32 \mathrm{GeV}^{2}$ [47]. Middle: $E_{0}=1.30 \mathrm{GeV}, \theta_{e}=37.5^{\circ}$, and $Q_{\mathrm{QE}}^{2} \approx 0.54 \mathrm{GeV}^{2}$ [47]. Bottom: $E_{0}=2.22 \mathrm{GeV}, \theta_{e}=15.5^{\circ}$, and $Q_{\mathrm{QE}}^{2} \approx 0.33 \mathrm{GeV}^{2}$ [48]. Black points show the data, solid black lines show the total GENIE prediction, colored lines show the contribution of the different reaction mechanisms: blue, QE; red, MEC; green, RES; and orange, DIS. 


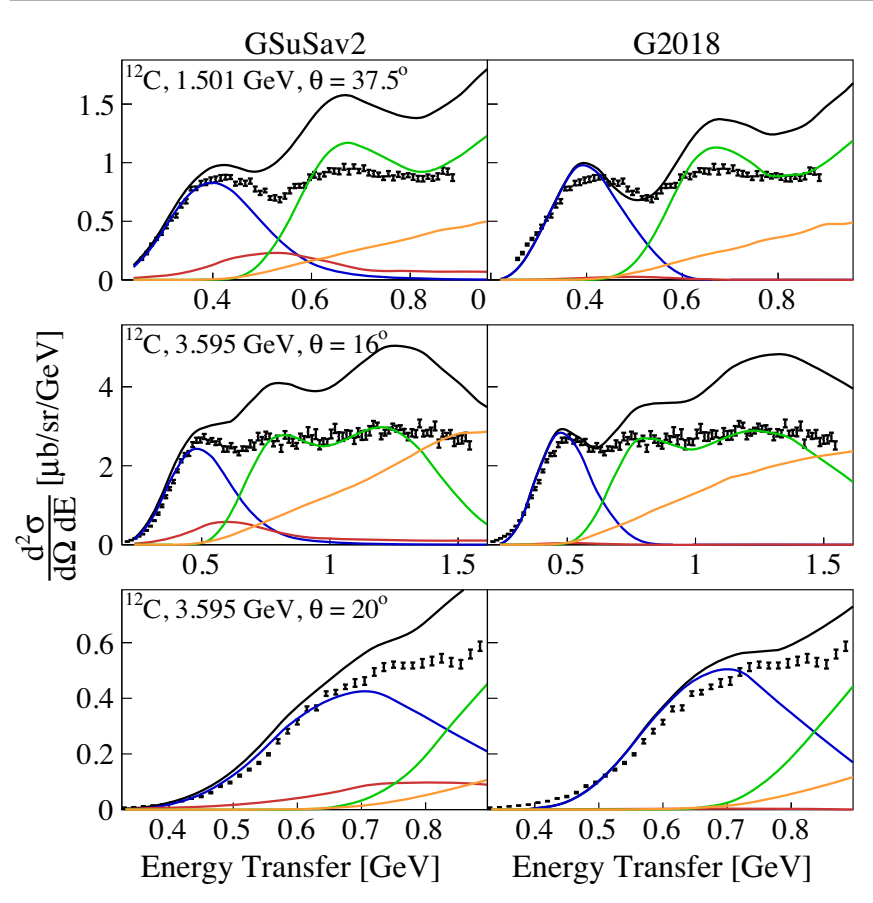

FIG. 8. Comparison of inclusive $\mathrm{C}\left(e, e^{\prime}\right)$ scattering cross sections for data and for GENIE. Left: data vs GSuSAv2. Right: data vs G2018. Top: $E_{0}=1.501 \mathrm{GeV}, \theta_{e}=37.5^{\circ}$, and $Q_{\mathrm{QE}}^{2} \approx 0.92 \mathrm{GeV}^{2}$ [47]. Middle: $E_{0}=3.595 \mathrm{GeV}, \theta_{e}=16^{\circ}$, and $Q_{\mathrm{QE}}^{2} \approx 1.04 \mathrm{GeV}^{2}$ [49]. Bottom: $E_{0}=3.595 \mathrm{GeV}, \theta_{e}=20^{\circ}$, and $Q_{\mathrm{QE}}^{2} \approx 1.3 \mathrm{GeV}^{2}$ [49]. Black points show the data, solid black lines show the total GENIE prediction, colored lines show the contribution of the different reaction mechanisms: blue, QE; red, MEC; green, RES; and orange, DIS.

contribution for G2018 appears to be much too small for $E_{0}=2.222 \mathrm{GeV}$ and $\theta_{e}=15.54^{\circ}\left(Q_{Q E}^{2}=0.33 \mathrm{GeV}^{2}\right)$. Both model sets significantly disagree with the data in the resonance region (where they use the same RES and DIS models). The $0.961 \mathrm{GeV}, 37.5^{\circ}$ and the $2.222 \mathrm{GeV}$, $15.54^{\circ}$ data are taken at almost identical $Q_{Q E}^{2}$. The lower beam-energy data are more transverse (since it is at larger scattering angle). The GSuSAv2 MEC contribution is similar for both datasets, but the G2018 MEC contribution is far smaller for the higher beam-energy data. The GSuSAv2 MEC contribution describes the dip region better in the higher beam-energy dataset. The RES model appears to agree with the data slightly better for the lower beamenergy, more transverse, dataset.

At the highest momentum transfers $\left(Q^{2} \approx 1 \mathrm{GeV}^{2}\right)$, the disagreement at the larger energy transfers is far greater. The G2018 "empirical" MEC model contributions are negligible, in marked contrast to the GSuSAv2 MEC contributions. The RES and DIS contributions are very significant at high $Q^{2}$ and in general the GENIE model is larger than the data in the region dominated by RES interactions, as noted in Ref. [16]. In addition, GENIE does not include the nuclear medium-dependent $\Delta$-peak shift, so

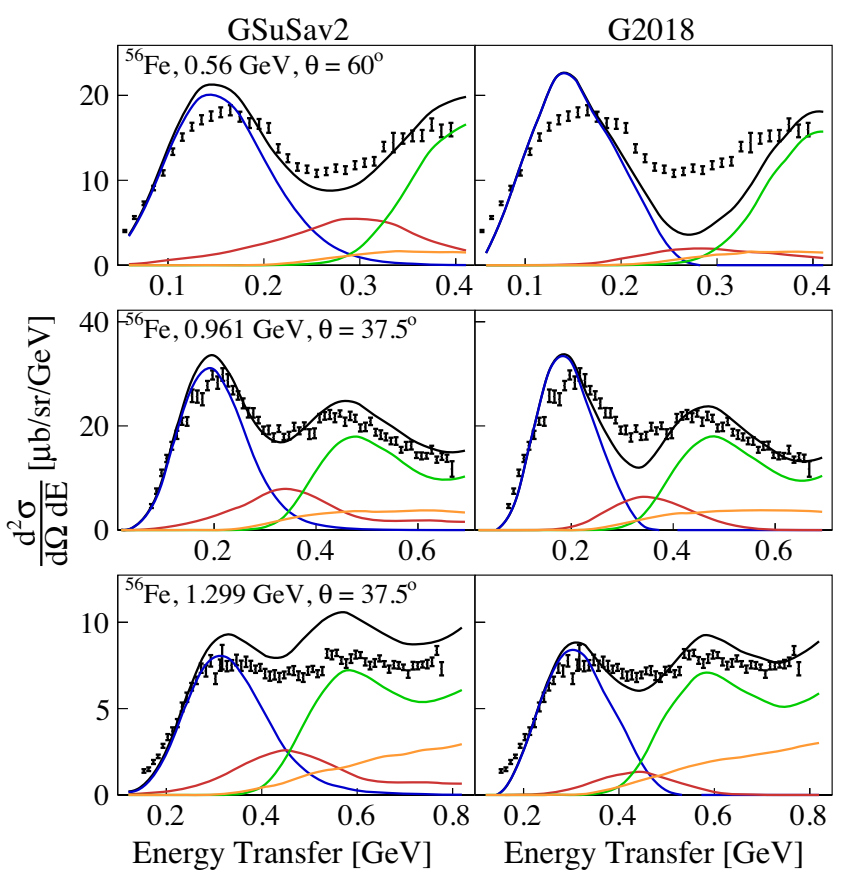

FIG. 9. Comparison of inclusive $\mathrm{Fe}\left(e, e^{\prime}\right)$ scattering cross sections for data and for GENIE. Left: data vs GSuSAv2. Right: data vs G2018. Top: $\mathrm{Fe}\left(e, e^{\prime}\right), E_{0}=0.56 \mathrm{GeV}, \theta_{e}=60^{\circ}$, and $Q_{\mathrm{QE}}^{2} \approx 0.24 \mathrm{GeV}^{2}$ [17]. Middle: $\mathrm{Fe}\left(e, e^{\prime}\right), \quad E_{0}=0.96 \mathrm{GeV}$, $\theta_{e}=37.5^{\circ}$, and $Q_{\mathrm{QE}}^{2} \approx 0.32 \mathrm{GeV}^{2}$ [47]. $\operatorname{Bottom:~} \mathrm{Fe}\left(e, e^{\prime}\right), E_{0}=$ $1.30 \mathrm{GeV}, \theta_{e}=37.5^{\circ}$, and $Q_{\mathrm{QE}}^{2} \approx 0.54 \mathrm{GeV}^{2}$ [47]. Black points show the data, solid black lines show the total GENIE prediction, colored lines show the contribution of the different reaction mechanisms: blue, QE; red, MEC; green, RES; and orange, DIS.

that the predicted location of the $\Delta$ peak is at larger energy transfer than that of the data.

Figure 9 shows the inclusive $\operatorname{Fe}\left(e, e^{\prime}\right)$ cross sections for several beam energies and scattering angles compared to the G2018 and GSuSAv2 models. The GSuSAv2 model describes the QE region better for all three datasets. As described in the section on modeling, the GSuSAv2 MEC model is independently calculated. The empirical model was fit using GENIE v2 QE and RES models; the fit will have to be redone once the QE and RES models stabilize. The GSuSAv2 MEC contributions are significantly larger than the empirical G2018 MEC contributions and match the dip-region data far better at $Q_{\mathrm{QE}}^{2}=0.24$ and $0.32 \mathrm{GeV}^{2}$. However, it overpredicts the dip-region cross section at $Q_{\mathrm{QE}}^{2}=0.54 \mathrm{GeV}^{2}$. The RES and DIS models describe the Fe data better than the $\mathrm{C}$ data at large energy transfers.

Figure 10 shows the inclusive $\operatorname{Ar}\left(e, e^{\prime}\right)$ cross sections for $E_{0}=2.222 \mathrm{GeV}$ and $\theta_{e}=15.54^{\circ}$ [50] compared to the G2018 and GSuSAv2 models. The GSuSAv2 model reproduces the data very well in the QE-peak region and the G2018 reproduces the data moderately well. The GSuSAv2 MEC model describes the dip region much 


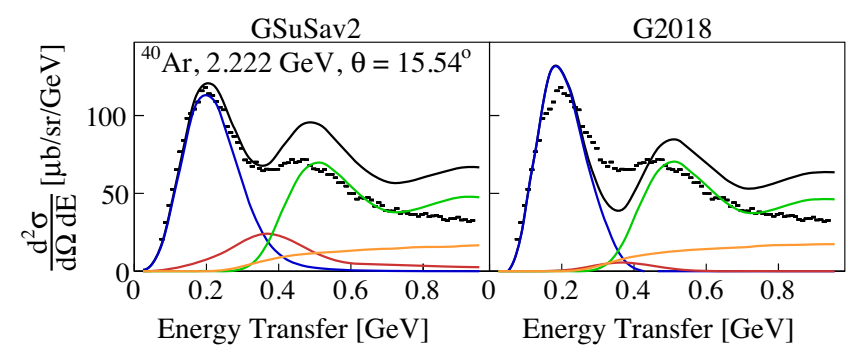

FIG. 10. Comparison of inclusive $\operatorname{Ar}\left(e, e^{\prime}\right)$ scattering cross sections for data and for GENIE at $E_{0}=2.22 \mathrm{GeV}, \theta_{e}=15.5^{\circ}$, and $Q_{\mathrm{QE}}^{2} \approx 0.33 \mathrm{GeV}^{2}$ [50]. Left: data vs GSuSAv2. Right: data vs G2018. Black points show the data, solid black lines show the total GENIE prediction, colored lines show the contribution of the different reaction mechanisms: blue, QE; red, MEC; green, RES; and orange, DIS.

better than the G2018 model. Again, there is significant disagreement with the RES and DIS models at larger energy transfers.

The quality of the agreement between data and GENIE depends more on the beam energy and angle than on the target mass (from $\mathrm{C}$ to $\mathrm{Fe}$ ). There is a possible momentumtransfer-dependent shift in the location of the SuSAV2 QE peak in $\mathrm{Fe}$ due to the extrapolation (via scaling) from $\mathrm{C}$ to $\mathrm{Fe}$.

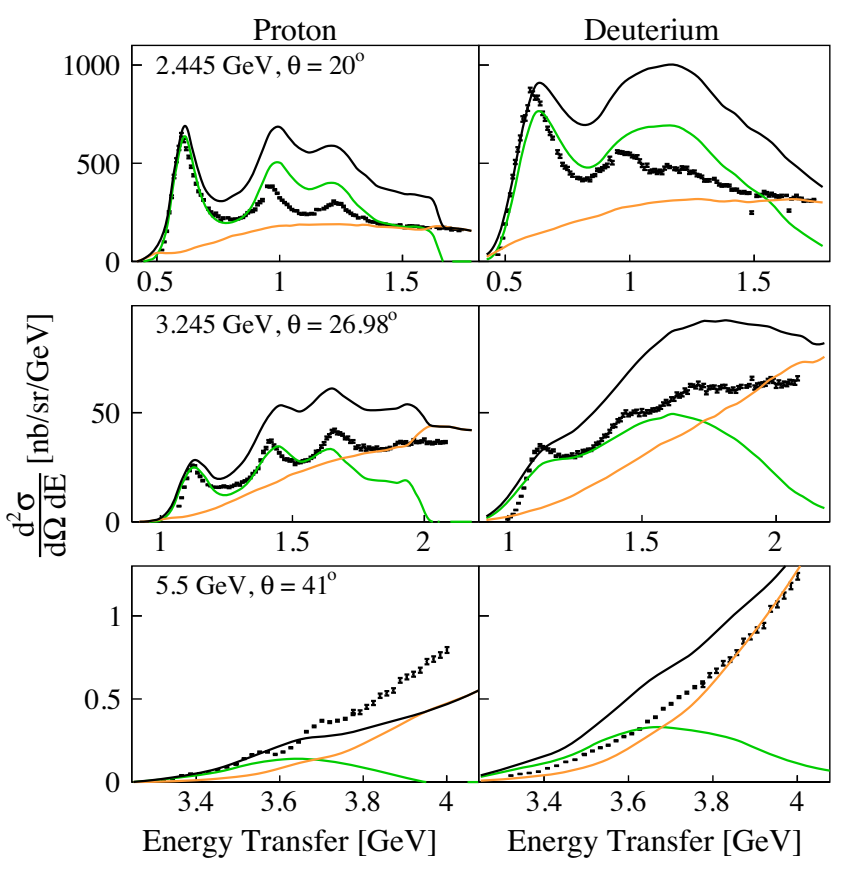

FIG. 11. Comparison of inclusive proton (left) and deuterium (right) $\left(e, e^{\prime}\right)$ scattering cross sections for data and for GENIE using G2018. Top: $E_{0}=2.445 \mathrm{GeV}$ and $\theta_{e}=20^{\circ}$. Middle: $E_{0}=$ $3.245 \mathrm{GeV}$ and $\theta_{e}=26.98^{\circ}$. Bottom: $E_{0}=5.5 \mathrm{GeV}$ and $\theta_{e}=$ $41^{\circ}$ [51-53]. Black points show the data, solid black lines show the total GENIE prediction, colored lines show the contribution of the different reaction mechanisms: green, RES; orange, DIS. The first peak at lowest energy transfer is the $\Delta(1232)$ resonance.
The GSuSAv2 QE model generally describes the data as well as or better than the G2018 model. The GSuSAv2 MEC model appears to be significantly superior to the empirical MEC model, especially at $Q^{2}<0.5 \mathrm{GeV}^{2}$ or at smaller scattering angles. The empirical MEC contribution is often much smaller than needed to explain the dip-region cross section. However, as an empirical model, it can be tuned to better describe the data.

$e$-GENIE dramatically overpredicts the large-energy transfer data at higher momentum transfers $\left(Q^{2}>\right.$ $0.5 \mathrm{GeV}^{2}$ ), indicating issues with the RES (BergerSehgal) and DIS (Bodek and Yang) models used.

This discrepancy at larger momentum and energy transfers is due to the elementary electron-nucleon cross section in the resonance and DIS regions, rather than to the nuclear models, since $e$-GENIE also significantly overpredicts the proton and deuteron cross sections, especially above the $\Delta$ peak (see Figs. 11 and 12). This shows that tuning the RES and DIS models to neutrino data [42] is not sufficient to constrain the vector part of the cross section.

\section{IMPLICATIONS FOR NEUTRINOS}

Electron-scattering data can be a very effective tool for testing neutrino event generators due to the similarity

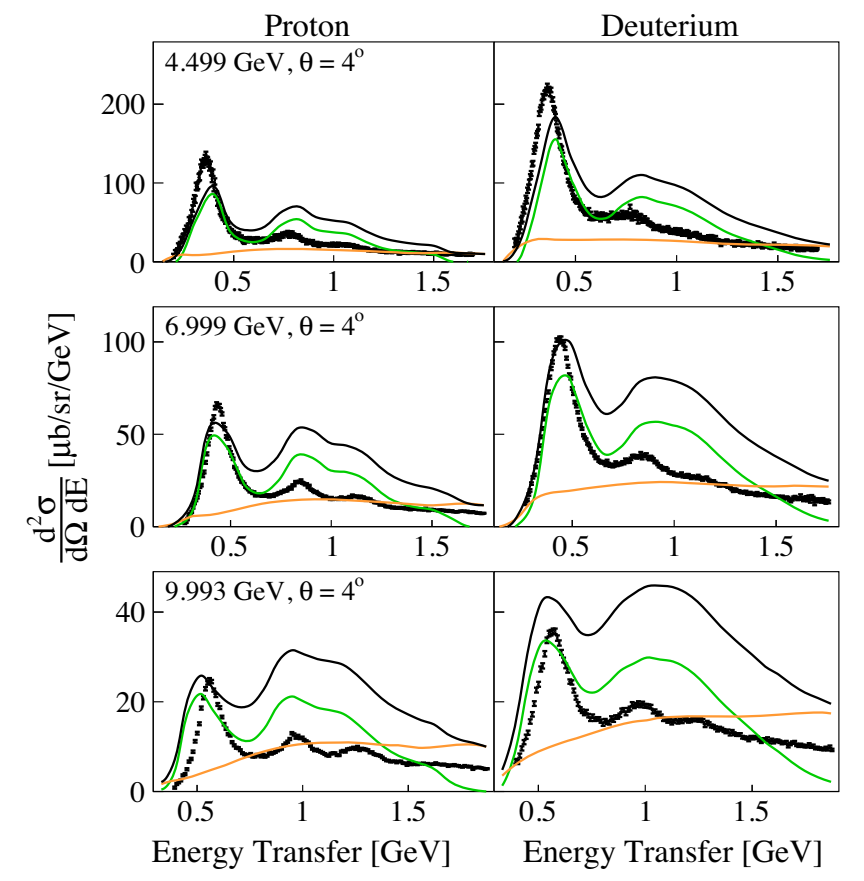

FIG. 12. Comparison of inclusive proton (left) and deuterium (right) $\left(e, e^{\prime}\right)$ scattering cross sections for data and for GENIE using G2018. Top: $E_{0}=4.499 \mathrm{GeV}$ and $\theta_{e}=4^{\circ}$. Middle: $E_{0}=$ $6.699 \mathrm{GeV}$ and $\theta_{e}=4^{\circ}$. Bottom: $E_{0}=9.993 \mathrm{GeV}$ and $\theta_{e}=4^{\circ}$ [54]. Black points show the data, solid black lines show the total GENIE prediction, colored lines show the contribution of the different reaction mechanisms: green, RES; orange, DIS. The first peak at lowest energy transfer is the $\Delta(1232)$ resonance. 

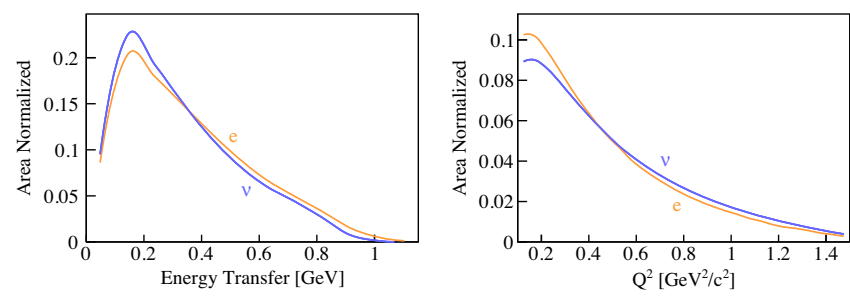

FIG. 13. Comparison of semiexclusive $1.16 \mathrm{GeV}$ lepton-carbon scattering for $Q^{2} \geq 0.1 \mathrm{GeV}^{2}$. The number of generated events is plotted versus energy transfer (left) and four-momentum transfer squared (right) for events with exactly one proton with $P_{p} \geq 300 \mathrm{MeV} / \mathrm{c}$, no charged pions with $P_{\pi} \geq 70 \mathrm{MeV} / \mathrm{c}$ and no neutral pions or photons of any momentum for $e$-GENIE electrons (orange) and GENIE CC $\nu_{\mu}$ (blue). The electron events have been weighted by $Q^{4}$. Both curves are area normalized.

between the interactions. Figure 13 shows the remarkably similar cross section shapes for electron-nucleus and neutrino-nucleus scattering for semiexclusive $1.16 \mathrm{GeV}$ lepton-carbon scattering with exactly one proton with $Q^{2} \geq$ $0.1 \mathrm{GeV}^{2}$ and $P_{p} \geq 300 \mathrm{MeV} / \mathrm{c}$, no charged pions with $P_{\pi} \geq 70 \mathrm{MeV} / \mathrm{c}$, and no neutral pions or photons of any momenta. This corresponds approximately to the Jefferson Lab CLAS detector thresholds. When comparing electron and neutrino distributions, the electron events are each weighted by $Q^{4}$ to reflect the difference in the electron and neutrino elementary interactions.
Exploiting these similarities within the same code is invaluable for minimizing the systematic uncertainties of future high-precision neutrino-oscillation experiments. Oscillation-analysis uncertainties exceeding $1 \%$ for signal and 5\% for backgrounds may substantially degrade the experimental sensitivity to $C P$ violation and mass hierarchy [3]. Such uncertainties include uncertainties in the $\nu$-nucleus interaction. These uncertainties are typified by the choices of the nuclear model available in GENIE.

Figure 14 shows that there is a larger difference among QE scattering models than there is between QE electron and neutrino scattering using the same nuclear model. All six panels show a "ridge," a maximum in the cross section as a function of energy transfer and momentum transfer. The length of the ridge (the decrease in intensity as the energy and momentum transfers increase) reflects the momentum-transfer dependence of the nucleon form factors used in the cross section model. The width of the distribution perpendicular to the ridge reflects the width of the nuclear momentum distribution. The momentum distribution of the local Fermi gas model cuts off at about $260 \mathrm{MeV} / \mathrm{c}$ for $\mathrm{C}$, whereas the relativistic mean field and the relativistic Fermi gas models have "tails" that extend to much larger momenta (see Fig. 15). The Nieves cross section decreases more slowly with momentum transfer than the others. For GSuSAv2, the electron cross section appears to decrease slightly faster with momentum transfer

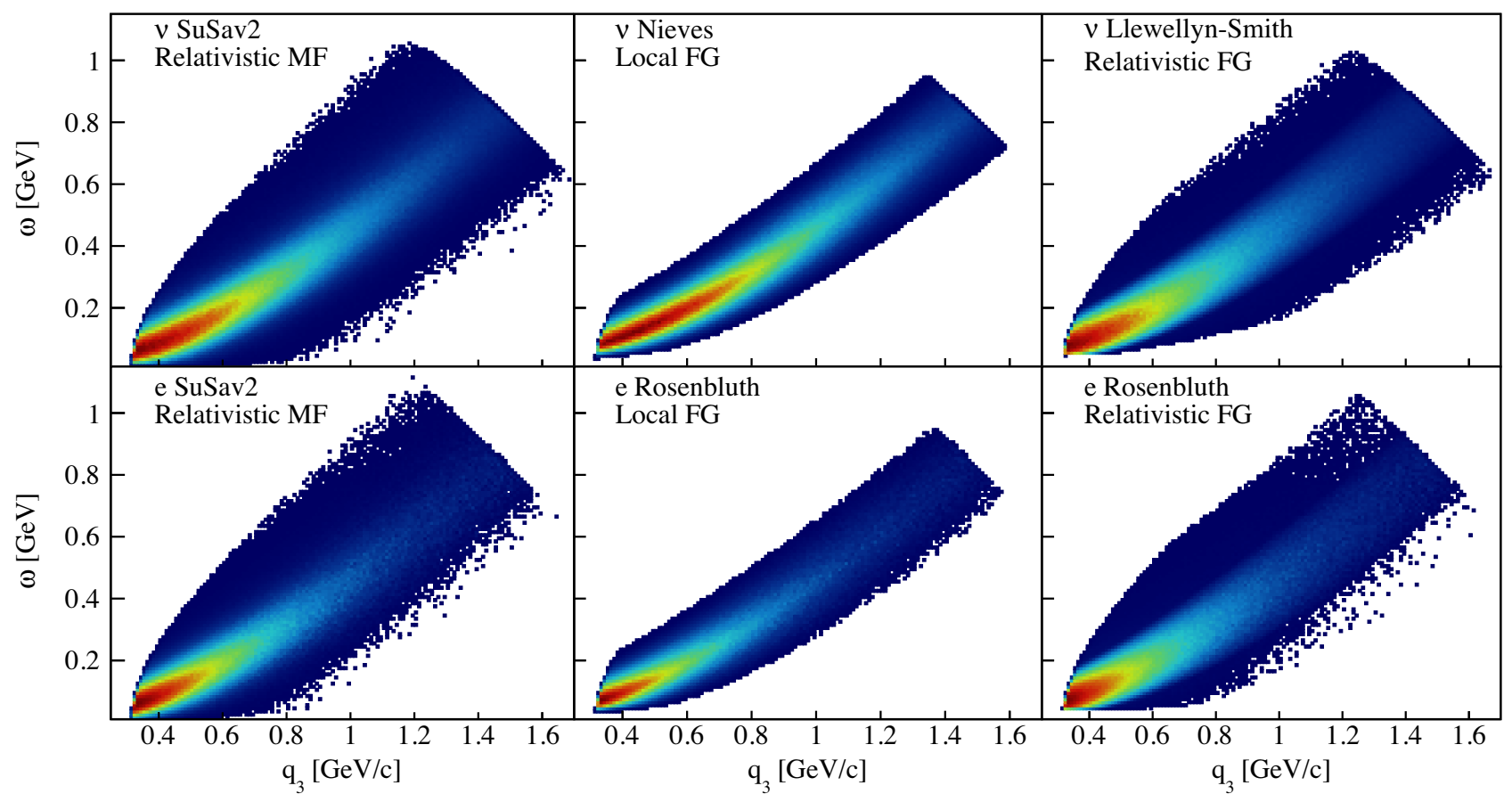

FIG. 14. Number of simulated events for QE scattering on ${ }^{12} \mathrm{C}$ at $1.161 \mathrm{GeV}$ with $Q^{2} \geq 0.1$ shown as a function of the energy transfer $\omega$ and the momentum transfer $q_{3}=|\vec{q}|$ for all the available nuclear models in GENIE for neutrinos (top) and for electrons (bottom). Left: the GSuSAv2 model which uses a relativistic mean field (RMF) momentum distribution. Middle: the Nieves or Rosenbluth cross section with the local Fermi gas (LFG) momentum distribution. Right: the Llewellyn-Smith or Rosenbluth cross section with the relativistic Fermi gas momentum distribution. The electron events have been weighted by $Q^{4}$. 


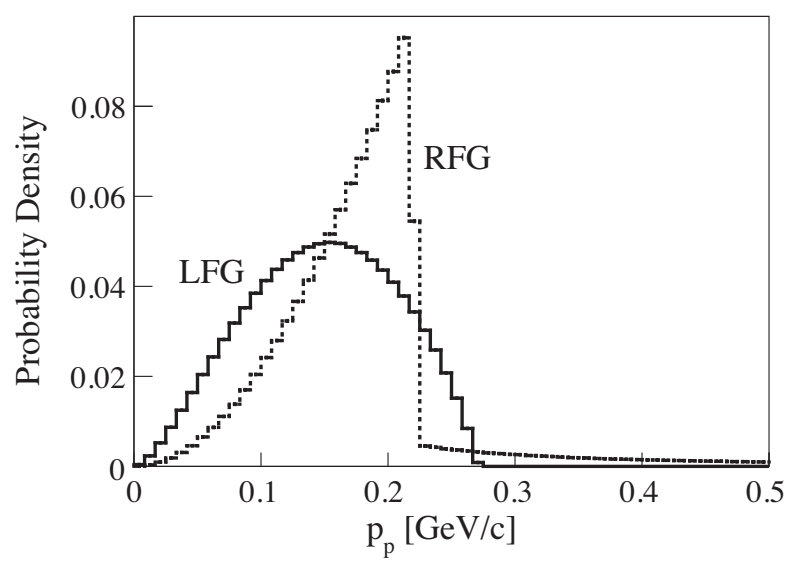

FIG. 15. Initial momentum distribution of protons in simulated QE C $\left(e, e^{\prime} p\right)$ events at $E=1.161 \mathrm{GeV}$ for the local Fermi gas (solid histogram) and relativistic Fermi gas (dotted histogram) models. The two curves are normalized to have the same area.

than the neutrino cross section, possibly reflecting differences in the axial and vector nucleon form factors. We compared the SuSAv2 and Rosenbluth/LFG models to electron scattering cross sections in the previous sections of this paper.

Similarly, Fig. 16 shows that the distribution of MEC events is very similar for electrons and for neutrinos within the same model. Thus, measurements of electron scattering will be able to significantly constrain models of neutrino scattering.

Our ability to use the GENIE code to transfer knowledge gained from electron scattering depends critically on the implementation of its components. Because of its modular design, all reaction models in GENIE use the same nuclear model (e.g., RFG or LFG). Although the electron-scattering capability was added after the initial code release, many of the reaction models used electron-scattering data to construct the vector components of neutrino interactions. This was true for the resonance [24,39] and the DIS [25] interactions. The difference between vector neutrino and electron scattering is an overall factor [see Eq. (4)] and an appropriate change in form factors.

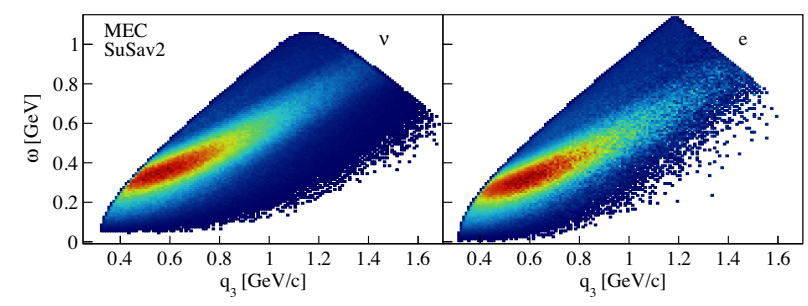

FIG. 16. Number of simulated events as a function of the energy transfer $\omega$ and of the momentum transfer $q_{3}=|\vec{q}|$ for neutrinos (left) and for electrons (right) using GSuSav2 for MEC interactions. The electron events have been scaled by $Q^{4}$ and all the samples have been generated with $Q^{2} \geq 0.1$.
Both QE and MEC models use the same vector form factors for neutrino and for electron scattering. QE models can use nucleon form factors from electron scattering [26], but MEC models must calculate the form factors.

The GENIE nonresonant meson-production cross section (referred to as "DIS") comes from the Bodek-Yang model [25] for the full cross section which extends to $\pi \mathrm{N}$ threshold. The cross section is scaled in the resonance region so that it agrees with $\nu D$ data [42]. Since a single factor is used to fit the model to the neutrino data, the highquality $e p$ and $e D$ data will be poorly described. While the total neutrino cross section and some of the hadronic content of the final state are loosely constrained by the $\nu D$ data, the vector component of the models is poorly constrained.

The QE models describe the data reasonably well in the low-energy transfer region. Similarly, the largest energy transfer portions of Figs. 8 and 11 show a reasonable agreement between GENIE and data. However, at intermediate energy transfer, the resonance region modeling disagrees with the data for both nuclear and nucleon targets (as in Ref. [16]). This is due to the use of resonance form factors that are not up to date (RES) and the way the nonresonant contribution was modeled.

Improvements are in progress but are not simple and therefore not available at this time. A possible shortterm fix would be to include the $e p$ and $e D$ inclusive electron-scattering models of Bosted and Christy [55,56]. Alternatively, the vector resonant form factors could be updated using electroproduction data from Jefferson Lab and elsewhere. A fit to those data is available [57] and partially implemented in GENIE, but it does not include nonresonant scattering. A more comprehensive solution would be to use the recent dynamical coupled channels model [58,59] to simultaneously describe both resonant and nonresonant scattering of both electrons and neutrinos.

\section{SUMMARY}

We significantly improved and updated the electron version of GENIE, the popular neutrino-nucleus event generator. We also added partial radiative corrections for electron scattering. Improvements came from bug fixes and extensions to the QE, $2 p 2 h$, and $\Delta$ excitation models for G2018 and an addition of the SuSav2 model for QE and MECs. The RES and DIS models are almost identical to past implementations [5] with the main change coming from a retune to the $\nu D$ data [42].

We compared two different GENIE model sets to inclusive electron-scattering data for a wide range of targets, beam energies, and scattering angles. The G2018 and GSuSAv2 model sets differ in their description of QE and MEC scattering. The SuSAv2 model generally describes the data at the QE peak as well as or better than the G2018 model. The SuSAv2 model set describes the dip region in most of 
the datasets much better than the G2018, especially at lower momentum transfer or smaller electron-scattering angle.

At the highest momentum transfers, $e$-GENIE dramatically overpredicts the data at large energy transfer, indicating significant problems with the momentum-transfer dependence of the RES and DIS models used. This discrepancy at larger momentum transfer is due to discrepancies in the electron-proton and electron-deuteron cross section rather than to the nuclear models. This conclusion is similar to that of Ref. [16], but we explore the difference in more detail. Tuning the RES and DIS models to neutrino data alone is not sufficient. Including electron-scattering data will allow tuning GENIE to describe the vector part of the RES and DIS interaction more precisely.

We found that the shapes of the scattered-lepton energy and momentum-transfer distributions are remarkably similar for electrons and for neutrinos, when the electron events are each weighted by $Q^{4}$ to reflect the difference in the elementary lepton interactions. In addition, the differences among QE interaction models are significantly larger than the difference between electron-nucleus and neutrinonucleus scattering. This validates our use of electronnucleus scattering data to constrain neutrino-nucleus scattering models.

The long term goal is to rigorously test the vector current part of the lepton-nucleus interaction and to use that information to improve modeling of neutrino-nucleus interactions. More extensive and more exclusive electron-scattering datasets are becoming available and will be used in the future. Simultaneously, improvements in GENIE are ongoing. The QE and MEC models have been significantly improved with the theoretically inspired SuSAv2 models. Similar improvements are needed in the RES and DIS modeling. The combination of new, high-precision, electron-scattering data and modern interaction models in GENIE should significantly decrease the systematic uncertainties of future neutrino-oscillation experiments.

\section{ACKNOWLEDGMENTS}

The authors thank the U.S. Department of Energy for support under Awards No. DE-FG02-96ER40960, No. DESC0007914, and No. DE-SC0020240. G. D. M. acknowledges support from CEA, CNRS/IN2P3, and P2IO, France; by the European Union's Horizon 2020 Research and Innovation Programme under the Marie SklodowskaCurie Grant Agreement No. 839481; by the Spanish Ministerio de Economia y Competitividad and ERDF (European Regional Development Fund) under Contract No. FIS2017-88410-P, and by the Junta de Andalucia (Grants No. FQM160 and No. SOMM17/6105/UGR). A.P. acknowledges support from the Visiting Scholars Award Program of the Universities Research Association. This document was prepared by the $e 4 \nu$ Collaboration using the resources of the Fermi National Accelerator Laboratory (Fermilab), a U.S. Department of Energy, Office of Science, HEP User Facility. Fermilab is managed by Fermi Research Alliance, LLC (FRA), acting under Contract No. DE-AC02-07CH11359.
[1] M. A. Acero et al. (NO $\nu$ A Collaboration), Phys. Rev. Lett. 123, 151803 (2019).

[2] K. Abe et al. (T2K Collaboration), Phys. Rev. Lett. 121, 171802 (2018).

[3] B. Abi et al. (DUNE Collaboration), arXiv:1807.10334.

[4] L. Alvarez-Ruso et al., Prog. Part. Nucl. Phys. 100, 1 (2018).

[5] C. Andreopoulos, A. Bell, D. Bhattacharya, F. Cavanna, J. Dobson, S. Dytman, H. Gallagher, P. Guzowski, R. Hatcher, P. Kehayias, A. Meregaglia, D. Naples, G. Pearce, A. Rubbia, M. Whalley, and T. Yang, Nucl. Instrum. Methods Phys. Res., Sect. A 614, 87 (2010).

[6] B. Abi et al. (DUNE Collaboration), https://home.fnal.gov/ ljf26/DUNEFluxes/.

[7] T. Katori and M. Martini, J. Phys. G 45, 013001 (2018).

[8] J. Amaro, M. Barbaro, J. Caballero, R. Gonzlez-Jimnez, G. Megias, and I. Ruiz Simo, J. Phys. G 47, 124001 (2020).

[9] O. Hen, G. A. Miller, E. Piasetzky, and L. B. Weinstein, Rev. Mod. Phys. 89, 045002 (2017).

[10] T. Katori, Meson exchange current (mec) models in neutrino interaction generators, arXiv:1304.6014.
[11] I. Ruiz Simo, J. Amaro, M. Barbaro, A. De Pace, J. Caballero, and T. Donnelly, J. Phys. G 44, 065105 (2017).

[12] K. Gallmeister, U. Mosel, and J. Weil, Phys. Rev. C 94, 035502 (2016).

[13] O. Buss, T. Gaitanos, K. Gallmeister, H. van Hees, M. Kaskulov, O. Lalakulich, A. Larionov, T. Leitner, J. Weil, and U. Mosel, Phys. Rep. 512, 1 (2012).

[14] S. Dolan, U. Mosel, K. Gallmeister, L. Pickering, and S. Bolognesi, Phys. Rev. C 98, 045502 (2018).

[15] G. Megias, J. Amaro, M. Barbaro, J. Caballero, T. Donnelly, and I. Ruiz Simo, Phys. Rev. D 94, 093004 (2016).

[16] A. M. Ankowski and A. Friedland, Phys. Rev. D 102, 053001 (2020).

[17] P. Barreau et al., Nucl. Phys. A402, 515 (1983).

[18] P. Bosted and V. Mamyan, arXiv:1203.2262.

[19] S. Dytman, Y. Hayato, R. Raboanary, J. Sobczyk, J. Tena Vidal, and N. Vololoniaina, arXiv:2103.07535.

[20] C. Andreopoulos, C. Barry, S. Dytman, H. Gallagher, T. Golan, R. Hatcher, G. Perdue, and J. Yarba, arXiv:1510 .05494 . 
[21] O. Hen, L. B. Weinstein, E. Piasetzky, G. A. Miller, M. M. Sargsian, and Y. Sagi, Phys. Rev. C 92, 045205 (2015).

[22] T. Katori, AIP Conf. Proc. 1663, 030001 (2015).

[23] S. Dolan, G. D. Megias, and S. Bolognesi, Phys. Rev. D 101, 033003 (2020).

[24] C. Berger and L. Sehgal, Phys. Rev. D 76, 113004 (2007).

[25] A. Bodek and U. K. Yang, J. Phys. G 29, 1899 (2003).

[26] R. Bradford, A. Bodek, H. S. Budd, and J. Arrington, Nucl. Phys. B, Proc. Suppl. 159, 127 (2006).

[27] J. Nieves, I. R. Simo, and M. J. V. Vacas, Phys. Rev. C 83, 045501 (2011).

[28] G. D. Megias, J. E. Amaro, M. B. Barbaro, J. A. Caballero, and T. W. Donnelly, Phys. Rev. D 94, 013012 (2016).

[29] J. Caballero, Phys. Rev. C 74, 015502 (2006).

[30] R. Gonzlez-Jimnez, A. Nikolakopoulos, N. Jachowicz, and J. Udas, Phys. Rev. C 100, 045501 (2019).

[31] R. Gonzlez-Jimnez, M. Barbaro, J. Caballero, T. Donnelly, N. Jachowicz, G. Megias, K. Niewczas, A. Nikolakopoulos, and J. Udas, Phys. Rev. C 101, 015503 (2020).

[32] C. Llewellyn Smith, Phys. Rep. 3, 261 (1972).

[33] J. Schwehr, D. Cherdack, and R. Gran, arXiv:1601.02038.

[34] A. De Pace, M. Nardi, W. Alberico, T. Donnelly, and A. Molinari, Nucl. Phys. A741, 249 (2004).

[35] I. Ruiz Simo, C. Albertus, J. Amaro, M. Barbaro, J. Caballero, and T. Donnelly, Phys. Rev. D 90, 033012 (2014).

[36] G. Megias et al., Phys. Rev. D 91, 073004 (2015).

[37] J. E. Amaro, M. B. Barbaro, J. A. Caballero, A. De Pace, T. W. Donnelly, G. D. Megias, and I. Ruiz Simo, Phys. Rev. C 95, 065502 (2017).

[38] R. P. Feynman, M. Kislinger, and F. Ravndal, Phys. Rev. D 3, 2706 (1971).

[39] D. Rein and L. Sehgal, Ann. Phys. (N.Y.) 133, 79 (1981).

[40] T. Yang, C. Andreopoulos, H. Gallagher, K. Hofmann, and P. Kehayias, Eur. Phys. J. C 63, 1 (2009).

[41] T. Sjöstrand, S. Mrenna, and P. Skands, J. High Energy Phys. 06 (2006) 026.

[42] J.Tena-Vidal et al. (GENIE Collaboration), Neutrinonucleon cross-section model tuning in GENIE v3, arXiv:2104 .09179 .
[43] S. Dytman and A. Meyer, AIP Conf. Proc. 1405, 213 (2011).

[44] S. G. Mashnik, A. J. Sierk, K. K. Gudima, and M. I. Baznat, J. Phys. Conf. Ser. 41, 340 (2006).

[45] Simc Monte Carlo, https://hallcweb.jlab.org/wiki/index .php/SIMC_Monte_Carlo, 2020.

[46] R. Cruz-Torres et al. (Jefferson Lab Hall A Tritium Collaboration), Phys. Lett. B 797, 134890 (2019).

[47] R. M. Sealock, K. L. Giovanetti, S. T. Thornton, Z.E. Meziani, O. A. Rondon-Aramayo, S. Auffret, J.-P. Chen, D. G. Christian, D. B. Day, J. S. McCarthy, R. C. Minehart, L. C. Dennis, K. W. Kemper, B. A. Mecking, and J. Morgenstern, Phys. Rev. Lett. 62, 1350 (1989).

[48] H. Dai et al. (Jefferson Lab Hall A Collaboration), Phys. Rev. C 98, 014617 (2018).

[49] D. Day et al., Phys. Rev. C 48, 1849 (1993).

[50] H. Dai et al. (The Jefferson Lab Hall A Collaboration), Phys. Rev. C 99, 054608 (2019).

[51] M. I. Niculescu, Inclusive resonance electroproduction data from hydrogen and deuterium and studies of quark-hadron duality, Ph.D. thesis, Hampton University, 2009.

[52] I. Niculescu et al., Phys. Rev. Lett. 85, 1186 (2000).

[53] S. P. Malace, Measurements of inclusive resonance cross sections for quark-hadron duality studies, Ph.D. thesis, Hampton University, 2006.

[54] S. Stein, W. B. Atwood, E. D. Bloom, R. L. A. Cottrell, H. DeStaebler, C. L. Jordan, H. G. Piel, C. Y. Prescott, R. Siemann, and R. E. Taylor, Phys. Rev. D 12, 1884 (1975).

[55] M. E. Christy and P. E. Bosted, Phys. Rev. C 81, 055213 (2010).

[56] P. E. Bosted and M. E. Christy, Phys. Rev. C 77, 065206 (2008).

[57] D. Drechsel, O. Hanstein, S. Kamalov, and L. Tiator, Nucl. Phys. A645, 145 (1999).

[58] H. Kamano, S. X. Nakamura, T. S. H. Lee, and T. Sato, Phys. Rev. C 94, 015201 (2016).

[59] S. X. Nakamura, H. Kamano, T. S. H. Lee, and T. Sato, AIP Conf. Proc. 1663, 070005 (2015). 